\title{
Owner Real-time Identification Scheme Based on Cycle Variation Law of Phone's Accelerometer
}

\author{
Heng Yin \\ ${ }^{1}$ Department of computer science \\ Zhengzhou Institute of Information Science and Technology, Zhengzhou 450001, China \\ yh_2846@163.com
}

Keywords: Smart phones, Gait, Identification, Accelerometer.

\begin{abstract}
A real-time identification scheme based on cycle variation law of android phone's accelerometer is proposed in this article to overcome the drawbacks of the current identification system such as simplicity, lag-behind in real-time responsiveness and low intelligence level. The implementation of this scheme includes three stages, namely owner feature data collection, feature analysis and identity recognition, where a feature extraction algorithm based on a kind of clustering idea is used for the identification. Various tests have demonstrated a high practicality of this scheme in real life usage due to a high accuracy result as much as $90 \%$ achieved in the tests.
\end{abstract}

\section{Introduction}

Along with the rapid development of the information society, performance of intelligent electronic equipment is getting better and better, more and more smart phones and other portable electronic equipment is becoming part of people's daily life and playing more important roles.

However, the greater the importance of smart phones become, the more severe the result will be to lose a phone, which is not only a direct economic loss of the phone itself but also the risk of disclosing the owner's accounts information. At present, the ways of unlocking a smart phone include password unlock, fingerprint identification unlock and hand gesture recognition unlock [1, 2], which enhances the security of the phone in certain degree. Though the common identity authentication schemes mentioned above are easy to operate, they cannot realize continuous and real-time identification and monitoring of user identity, and the recognition requires users to cooperate actively, and multiple verifications could reduce user experience as well. In addition, many methods of attack for the above schemes have appeared, resulting in a security reduction.

Above analysis concludes that it is necessary to design and realize an effective and real-time user identification scheme to safeguard user's phone security as well as improve user experience. The scheme proposed in this article is designed on the basis of the original security measures and is able to recognize user identity initiatively and enhance mobile security by continuous sensing users' motion features.

\section{Related Work}

\subsection{Phone User Identification}

Currently, a commonly used identification method is password authentication. However, no matter who is using the phone, whether he/she is the owner or not, as long as the password is matched, he/she is allowed the access to the phone, this is how certain potential safety risk exists.

Fingerprint identification technology combines both software and hardware. The hardware device scans and collects the fingerprint data and the software outputs the identification results. But once the 
fingerprint is captured, the impostors can unlock the phone easily and even make mobile payment, which leads to economic losses to the owner.

The above identification methods all require the users' proactive coordination for identification, consequently bring down user experiences. While individual gait features are difficult to duplicate and imitate, the wide-spread of smart phones and the precision improvement of its internal sensors make it easier to acquire one's behavioral features data and facilitates the realization of a real-time identification scheme based on cycle variation law of the accelerometer in the phone.

\subsection{Identification Based on Motion Features}

Nowadays, almost all smart phones are equipped with internal accelerometers. Identification based on motion features acquired from the accelerometers has become an important branch in the area of identification study, which includes data collection and pre-processing, data analysis and recognition, etc.[3] Data collection and pre-processing is to collect acceleration signals[4] generated by individual motions. Lying at the bottom of the entire system, it is the foundation to realize the motion features identification. Data analysis lies in between the pre-processing segment and the recognition segment, the function of which is to train the pre-processed data. Data analysis is an important segment of the motion features identification. Recognition segment involves several classification algorithms [5] such as Decision Tree, SVM (Support Vector Machine) and Bayes Classifier.

\section{The Proposed Scheme}

The identification scheme proposed in this article will adopt modular design concept, which includes data collection, data pre-processing, features learning and identification. The detailed flow chart is as shown in figure 1.

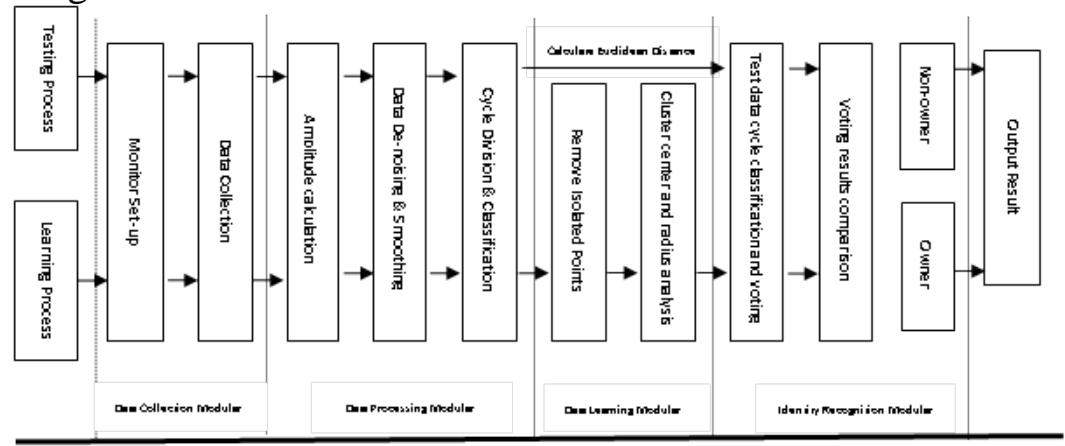

Fig. 1 System Flow Chart

Following are the detailed illustrations according to the system flow chart shown in Figure 1.

\subsection{Data Collection}

Mobile's accelerometer is able to sense the mobile's velocity change events and generate data stream comprising of accelerated velocities in the unit of gravity in three axes. Thus, data collection segment could invoke relative functions inside the java program and set up a category correlative with the accelerometer to monitor the events and continuously collect user's motion data, eventually provides data base for the motion feature recognition.

\subsection{Training}

Data pre-processing modular consists of acceleration amplitude value calculation, cycle sequence division and classification steps, the flow chart of which is as shown in figure 2.

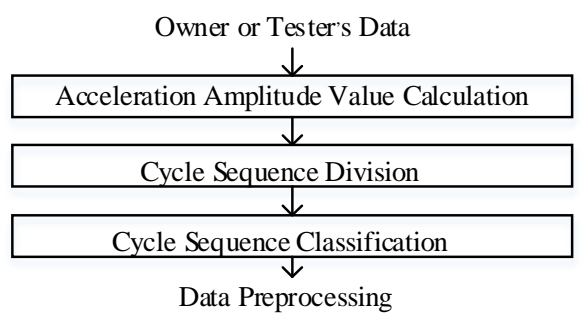

Fig. 2 Pre-processing Modular Flow Chart 
Detailed execution flows are as below:

Step1: Acceleration Amplitude Value Calculation

(1) Save data collected from the mobile's accelerometer in array X[count], Y[count] and Z[count], put the quantity number of data as the count variables.

(2) Calculate the acceleration amplitude value and save as array FU [count].

Step2: Cycle Sequence Division

(1) Go through array FU[count].

(2) Save low points of the minimal amplitude value in one cycle sequence in array low point[ ].

Step3: Classification of Cycle Sequence with Different Length

(1) Go through array low point[ ].

(2) $\mathrm{Ki}=$ low point[i]-low point [i-1].

(3) Classify ki.

(4) Record number of cycle sequences.

(5) Define flag identifier and identify whether cycle sequences exist in the two-dimensional array.

So far, cycle sequences with different length have been classified.

Each cycle sequence could be regarded as an abstract point in some dimensional space, we call it data point. Since data collected from improper actions to the phone such as improper operation of the screen or phone direction steered are very different from data collected from proper walk, we call such data as isolated point. In order to learn the owner motion features better, we must remove these isolated points to minimize their impacts.

Detailed execution flows are as below:

Step1: Calculate the center of the data points

(1) Use flag identifier to identify whether data points exist.

(2) If data points exist, calculate their center.

Step2: Calculate Distance D

(1) Use flag identifier to identify whether data points exist

(2) If data points exist, calculate average Euclidean Distance between data points and center, multiply the average by $\mathrm{M}$ to get $\mathrm{D}$ value.

Step3: Remove Isolated Points

(1) Use flag identifier to identify whether data points exist.

(2) If data points exist, calculate Euclidean Distance between data points and center.

(3) Compare numerical value of Euclidean Distance and D.

(4) If Euclidean Distance value is bigger than D, remove the isolated point, otherwise, retain this data point and keep going through all data points.

Step4: Decide Whether to Repeat the Above Steps

(1) Evaluate ratio of data points whose distances are below average to total data points is within $(0.5-\mathrm{K}, 0.5+\mathrm{K})$ range or not.

(2) If not, repeat Step 1, 2, 3 and 4 again; if yes, go to Step 5 directly.

Step5: Define cluster center and border radius

(1) Calculate center of the remaining data points as the final cluster center.

(2) Define the Euclidean Distance between cluster center and the farthest data point as the border radius.

\subsection{Identification}

Collect real-time data measured from the three axis of accelerometer in the specified time interval and make owner or non-owner judgment after algorithms. Detailed execution flows are as below:

Step1: Acquire owner feature data and tester data

(1) Acquire owner's center and border radius values from the data learning modular.

(2) Acquire tester's data points and corresponding flag identifier.

Step2: Compare numerical value of Euclidean Distance and border radius of each data point

(1) Use flag identifier to identify whether data points exist.

(2) If data points exist, calculate Euclidean Distance between data points and center. 
(3) Compare the Euclidean Distance and border radius and record the number of comparisons.

(4) Record number of data points with Euclidean Distance less than or equals to the border radius, regard them as number of normal data sequences.

Step3: Identity Voting

(1) Calculate ratio between number of normal data sequences and total number of data sequences.

(2) If the ratio is above or equals to the threshold value, it votes for the owner, otherwise, it votes for the non-owner.

\section{Testing}

\subsection{Owner Feature Learning}

Multiple tests have helped us find the optimal parameter setups for this identification scheme, which are to set $\mathrm{M}$ at 1.2, to set $\mathrm{K}$ at 0.1 , to specify learning time span and test time span at 10 seconds and to use Red Mi 1S as the test phone model. 34 students have been selected for a complete test to collect data and learn features. 4 students were selected as the owners and marked as user A (Male), User B (Male), User C (Female) and User D (Female). They were required to hold the phone in the right hand with screen facing inward and went through the data collection, feature abstraction and record steps.

\subsection{Owner Test}

Ask User A, B, C and D who have completed feature learning to hold the phone in right hand with screen facing inward and walk properly for 10 seconds, repeat 50 times and record the average results as shown in Table 1.

Table 1 Identification Results of Four Owners

\begin{tabular}{|c|c|c|c|c|}
\hline Owner & $\begin{array}{c}\text { Number of } \\
\text { Tests }\end{array}$ & $\begin{array}{c}\text { Number of Right } \\
\text { Identity }\end{array}$ & $\begin{array}{c}\text { Number of } \\
\text { Wrong Identity }\end{array}$ & $\begin{array}{c}\text { Accuracy } \\
\text { Rate }\end{array}$ \\
\hline A & 50 & 50 & 0 & $100 \%$ \\
\hline B & 50 & 49 & 1 & $98 \%$ \\
\hline C & 50 & 48 & 2 & $96 \%$ \\
\hline D & 50 & 50 & 0 & $100 \%$ \\
\hline Average & - & - & - & $98.5 \%$ \\
\hline
\end{tabular}

From the above owner test results, we could see the average accuracy rates of owner A, B, C and D are $100 \%, 98 \%, 96 \%, 100 \%$, which are over 95\%, which means this scheme provides a very high identification accuracy for the owner himself.

\subsection{Non-owner Test}

Ask the remaining 30 students to use the phone stored User A's features to test whether the scheme could recognize they are owner or not, considering influencing factors of their gender and height, etc.

Select 10 students from the remaining 30 students who have the same gender and similar height with User A, ask them to hold the phone in right hand with screen facing inward and walk properly for 10 seconds, repeat 50 times and record test results as shown in table 2 .

Table 2 Identification Results of Testing Users

\begin{tabular}{|c|c|c|c|c|c|}
\hline Testing User & Gender & $\begin{array}{c}\text { Number of } \\
\text { Testing }\end{array}$ & $\begin{array}{c}\text { Number of } \\
\text { Non-owner Results }\end{array}$ & $\begin{array}{c}\text { Number of } \\
\text { Owner Results }\end{array}$ & $\begin{array}{c}\text { Accuracy } \\
\text { Rate }\end{array}$ \\
\hline 1 & Male & 50 & 43 & 7 & 86 \\
\hline 2 & Male & 50 & 44 & 6 & 88 \\
\hline 3 & Male & 50 & 41 & 9 & 82 \\
\hline 4 & Male & 50 & 45 & 5 & 90 \\
\hline 5 & Male & 50 & 46 & 4 & 92 \\
\hline 6 & Male & 50 & 43 & 7 & 86 \\
\hline 7 & Male & 50 & 41 & 9 & 82 \\
\hline 8 & Male & 50 & 39 & 11 & 76 \\
\hline 9 & Male & 50 & 40 & 10 & 80 \\
\hline 10 & Male & 50 & 47 & 3 & 94 \\
\hline Average & -- & -- & -- & -- & $85.60 \%$ \\
\hline
\end{tabular}


The test results show a pretty well performance of the scheme with average accuracy achieving $85.6 \%$ for non-owner identification.

\section{Conclusions}

Aimed at further improving phone security, a real-time identification scheme based on the cycle variation law of phone's acceleration is proposed in this article. This scheme is able to realize real-time owner identity recognition and monitor through several steps including user data collection, data analysis and identity recognition, etc. A large number of tests have been run to verify the scheme's accuracy rate. And the test results conclude that high identification success rate and excellent user experience advantages bring this scheme certain practical value in securing owner's personal information and guarding phone theft.

\section{References}

[1] Li Yongmou -Technological Research on Smart Phone's Motion Recognition and Identification Changsha: Graduate School of National University of Defense Technology - 2014:1-55.

[2] Ma Jianping, Pan Junqing, Chen Bo - Hand Gesture Recognition Method of Android Smart Phones[J] - Mini-Micro Systems, Journal of Chinese Computer Systems - 2013:34(7):1703-1707.

[3] Xu Chuanlong - Human Behavior Recognition Based on 3-Dimensional Accelerometer Hangzhou: School of Information Engineering of Zhejiang University of Technology - 2013:1-69.

[4] Dong L, Zhang K. Sensing and Control of MEMS Accelerometers Using Kalman

filter[C]//Control and Decision (CCDC),2012 24th Chinese IEEE, 2012:3074-3079.

[5] Yang Tianqi - Artificial Intelligence and Its Application - Jinan University Press - June, 2014.

[6] Everitt B, Hothorn T. Cluster Analysis [M]//Cluster analysis. Wiley, 2011:115-136.

[7] Liang Jiuzhen, Zhu Xiangjun, Chen Jing - The Design of High Precision Low Sampling Algorithm Based on Mobile Accelerometer - Journal of Northwest University (Natural Science Edition)- 2015, 45(5):738-744.

[8] Chen Shouwen, Li Mingdong - Algorithm Implementation Based on K-means Object-oriented Idea [J] - Chuzhou College Journal - 2008, 10 (3):42-44.

[9] Song Haoran, Liao Wenshuai, Zhao Yiming - High Precision Pedometer Based on Accelerometer ADXL330 - Journal of Transduction Technology - 2006, Vol.19: 8-4.

[10] Zhu Yuanbo - Android Sensor Development and Intelligent Device Cases and Applications Posts \& Telecom Press - May, 2016. 